Proceedings

\title{
Identification of the Design Parameters for a Spacer Fabric Pressure-Mapping Sensor ${ }^{\dagger}$
}

\author{
Tianchen Shen 1,*, Samuel Pitou ${ }^{1}$, Ryo Eguchi ${ }^{2}$ and Matthew Howard ${ }^{1}$ \\ 1 Department of Engineering, King's College London, London WC2R 2LS, UK; Samuel.Pitou@kcl.ac.uk (S.P.); \\ matthew.j.howard@kcl.ac.uk (M.H.) \\ 2 School of Science for Open and Environmental Systems, Graduate School of Science and Technology, \\ Keio University, Yokohama 223-8522, Japan; eguchi.ryo@keio.jp (R.E.) \\ * Correspondence: Tianchen.Shen@kcl.ac.uk \\ + Presented at the International Conference on the Challenges, Opportunities, Innovations and Applications \\ in Electronic Textiles (E-Textiles 2020), Virtual venue, UK, 4 November 2020.
}

Citation: Shen, T.; Pitou, S.; Eguchi R.; Howard, M. Identification of Design Parameters for a Spacer Fabric Pressure Mapping Sensor. Proceedings 2021, 68, 15. https://doi.org/10.3390/ proceedings2021068015

Published: 21 January 2021

Publisher's Note: MDPI stays neutral with regard to jurisdictional claims in published maps and institutional affiliations.

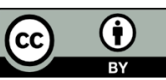

Copyright: (C) 2021 by the authors. Licensee MDPI, Basel, Switzerland. This article is an open access article distributed under the terms and conditions of the Creative Commons Attribution (CC BY) license (http://creativecommons.org/licenses/by/4.0/).

\begin{abstract}
This work presents an empirical study into the design of fabric pressure sensors, taking into account the electro-mechanical variability of spacer fabric. The saturation of the fabric sensor at high force levels is a major factor limiting the range of measurable pressures. In order to address this, experiments were carried out to investigate several methods of modifying the sensitivity characteristics through the layering and specifications of the textile sheets.
\end{abstract}

Keywords: electrical impedance tomography; conductive spacer fabric; pressure-mapping device

\section{Introduction}

Electronic textiles are often used for wearable devices because they are soft, lightweight, flexible, and breathable [1]. Their deformability, moreover, makes them ideal media for electrical impedance tomography (EIT), as it enables images to be formed that map the pressure and forces applied by the body to the wearable device. This has several applications, for instance, in the design of smart insoles to unobtrusively measure the features of gait and posture [2]. EIT is a functional imaging technology. In its use in e-textiles, the principle is to apply a weak current to a series of points around the conductive fabric, and to measure the resultant voltage value on electrodes distributed about its perimeter. Accordingly, the relationship between the voltage and the current is used to reconstruct the internal resistance [3]. Since, in many e-textiles, the latter depends on the deformation of the fabric, the resultant signal can be used to reconstruct the magnitude and location of the pressure or forces applied to the surface.

A challenge with the design of such fabric sensors, however, is matching the sensitivity with the requirements of the application. For instance, if the application requires the measurement of high pressure levels (e.g., the weight of an adult distributed over the surface area of an insole), the sensor may rapidly reach saturation, preventing the details of the pressure map appearing. Pressure mats and force plates feature high accuracy and show a pressure map within a large measurable range, but their method of installation is in-ground [4]. As such, they cannot be used for measurements in daily life. One of the main difficulties for EIT-based pressure mapping devices is the uncertainty in the exact relationship between the applied pressure and the conductivity of the fabric. This is particularly important when selecting the optimal textile substrate and the specification of the fabric, especially when considering textiles with complex structures, such as spacer fabric.

In order to address this issue, this paper proposes several methods to increase the measurable pressure range, and it presents a detailed analysis of the relationship between 
pressure and resistance for spacer fabric in order to identify the performance of it during the pressing process and find the optimal specification of the fabric. By taking the proposed approach, the maximum range of the measurable pressure increases by $300 \%$.

\section{Background}

\subsection{Length-Related Resistance and Contact Resistance}

In the fabrics considered in this study, the overall resistance of the fabric is the superposition of two types of resistance: (i) the length-related resistance, and (ii) the contact resistance. These influence the EIT-based pressure imaging during force-induced deformation [5]. The contact resistance dominates the total resistance initially, then the lengthrelated resistance takes over the contact resistance during the further stretching process [6].

The equation determining the relationship between the material properties and its resistance is

$$
R=\alpha\left(\rho \frac{\mathrm{L}}{\mathrm{A}}\right)+\beta\left(\frac{\rho}{2} \sqrt{\frac{\pi H}{\mathrm{nP}}}\right)
$$

where $R$ is the total resistance, $\mathrm{Q}$ is the resistivity, $\mathrm{H}$ is the material hardness, $\mathrm{P}$ is the pressure, $\mathrm{n}$ is the number of contact points, $\mathrm{L}$ is the length of the specimen, $\mathrm{A}$ is the crosssection area of the specimen and $\alpha$, and $\beta$ describe weights of two resistances during the stretching process respectively. In these parameters, $\mathrm{H}, \mathrm{n}$ and $\mathrm{Q}$ are constant, and depend on the material. Conversely, $\alpha, \beta, \mathrm{L}$ and $\mathrm{A}$ are variable during the pressing process. The first part of Equation 1 represents the length-related resistance, and the last part is the contact resistance.

\subsection{The Change in Resistance of Conductive Fabric during the Stretching Process}

According to [7], the evolution of the surface resistance of spacer fabrics can be decomposed into three regions, depending on the strain applied. The first region is a resistance-decreasing region in which the contact resistance is prevalent: while $\mathrm{n}, \mathrm{L}$ and $\mathrm{P}$ increase, A decreases when pressure is applied to the fabric. Therefore, the total resistance becomes lower when a low strain is applied. The second region is a stationary region in which the contact- and length-related resistance are stable. The third region is reached when high strain is applied. In this region, the length-related resistance causes the total resistance to increase with continued stretching. In the application considered in this paper (namely, pressure sensing), the fabric is always pressed, rather than stretched, so the third region is rarely seen. Therefore, the remainder of this paper focuses on the first region of the sensor response.

\section{Material and Methods}

\subsection{Spacer Fabric Pressure Mapping Sensor}

The ideal EIT-based pressure mapping sensor should generate its conductive area by the use of conductive yarns in a garment, preferably in a knitted structure. [3] Therefore, spacer fabric is a suitable material for an EIT-based wearable device, since spacer fabrics are three dimensional fabrics created by the connection of two flat knitted fabrics with spacer yarns or microfilaments. The fabric sensor used in this study was developed by Statex Productions and Vertriebs Gmbh, Germany (Shieldex Spacer Fabric B). It was used with an average surface resistivity below $1 \Omega / \mathrm{m}^{2}[8]$.

\subsection{Experiment Setup}

Figure 1a shows the overview of the system. There are 16 electrodes spread equally around the boundary of the spacer fabric, and it has a substrate (white foam board) underneath the fabric. When a weight is put onto the fabric, it can reconstruct the pressure information and show it on a laptop. 


\subsection{Protocol}

The first step of the experiment is to prepare several circular weights to be placed on the spacer fabric. Circular weights were chosen because they allow the point with the highest pressure to be found by seeking their geometrical centre. Then, the weights are placed at a fixed point on the fabric, and the total resistance of the fabric within the contact area can be recorded according to the corresponding signal value (the total resistance of the fabric) from the reconstructed pressure map. In order to avoid the problem of hysteresis, an interval of $30 \mathrm{~s}$ is given between the placement of each weight and the recording of the reconstructed image. Then, the weight is gradually increased until the signal value is saturated (i.e., it ceases to change). In the experiment below, the results were reported for 10 repetitions of this procedure. MATLAB (MathWorks company, USA) was used to reconstruct the pressure information, and the EIDORS platform provided the necessary forward and inverse EIT algorithms.

\section{Experiments}

\subsection{Experiment 1}

In order to investigate the effect of the hardness of the substrate on the measurable pressure range, Experiment 1 used soft foam board (Universal Seating Dining Seat Stool Chair Foam Square, Upholstery, MC- CASES Company, Germany) and hard foam board (UCREATE foam board, Pacon Corp, USA) as the substrate.

\subsection{Experiment 2}

In order to state the exact relationship between the pressure and resistance of the spacer fabric, and whether the theory in the background can be used for the spacer fabric (Shieldex Spacer Fabric B, Germany), in this experiment, Equation 1 was used to calculate the total resistance as a theoretical result This experiment used hard foam board (UCREATE foam board, Pacon Corp, USA) as the substrate, in order to avoid changing the pressing deformation. It can thus show whether the experimental results and theoretical results are matched. If they are matched, Equation 1 is useful to find the optimal specifications (i.e., resistivity, hardness, etc.) of the spacer fabric.

\section{Results}

\subsection{Experiment 1}

Figure $1 \mathrm{~b}$ shows that soft foam board has a lager measurable pressure range. When the fabric is mounted on a hard substrate, the gradient of the curve is steeper, indicating greater sensitivity; however, saturation is reached at a much lower pressure level. The softer substrate, however, allows for a greater measurable range, in which saturation is reached only after around $7 \mathrm{kPa}$. The main reason is that the use of hard and soft foam board cause two different pressing deformations of the fabric, as shown in Figure 1c.

\subsection{Experiment 2}

Figure $1 \mathrm{~d}$ shows that the theoretical and experimental results are in close agreement. Figure 1e shows that contact resistance dominates the total resistance initially, and that length-related resistance takes over during further pressing processes. The theoretical results were calculated by Equation 1. In this equation, $\mathrm{n}$ (the density of the conductive yarns) and $\varrho$ (the resistivity) were obtained from the datasheet. $\mathrm{H}$ (the hardness of the material) was measured by the tester (LX-C Material hardness tester, Almencla company, USA). According to Equation 1, higher resistivity, the lower density of conductive yarns, and stiffer spacer fabric can increase the measurable pressure range. 


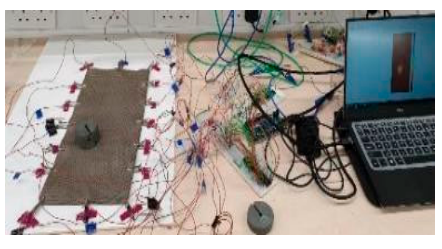

(a)

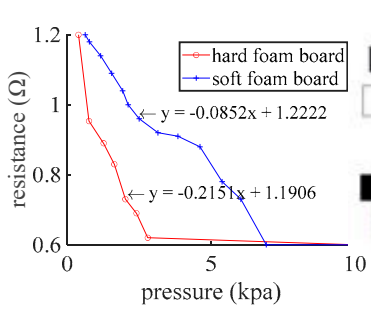

(b)

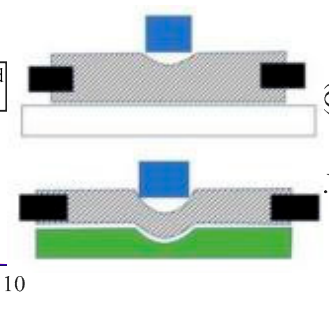

(c)

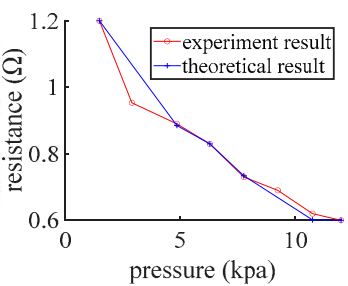

(d)

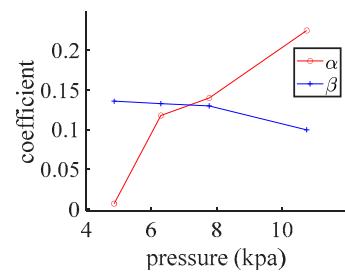

(e)

Figure 1. (a) The overview of the system (top: weights, middle: spacer fabric; bottom: substrate; right: laptop); (b) Experiment 1 results; (c) the side view of the different pressing deformations (white rectangle: hard foam board, green rectangle: soft foam board); (d) Experiment 2 results; (e) $\alpha, \beta$ during pressing process.

\section{Conclusions}

In this paper, several methods to increase the measurable pressure range of an EITbased pressure mapping fabric sensor were presented. The first method is to use a softer substrate in order to change the pressing defamation of the conductive fabric. The second method is to combine a higher resistivity fabric (i.e., with a lower density of conductive yarns) and a stiffer spacer fabric from the analysis of the relationship between the pressure and resistance. From my own perspective, the best method is to use soft foam board as the substrate, low density conductive yarns, and stiffer fabric, since this is the most convenient and the most direct approach to increase the measurable pressure range.

Author Contributions: T.S. designed and carried out the experiments and data analysis, and wrote the manuscript draft. S.P. and E.R. helped T.S. to analyze the data. M.H. supervised all of the research work and edited the manuscript. All authors have read and agreed to the published version of the manuscript.

Funding: This research received no external funding.

Institutional Review Board Statement: N/A.

Informed Consent Statement: 'Not applicable' for studies not involving humans.

Data Availability Statement: All relevant data are publicly available through the following URL: http://doi.org/doi:10.18742/RDM01-697.

Conflicts of Interest: The authors declare no conflict of interest.

\section{References}

1. Çiçek, M. Wearable technology and its future application. Int. J. Electr. Electr. Data Commun. 2015, 3, $2320-2084$.

2. Russo, S.; Nefti-Meziani, S.; Carbonaro, N.; Tognetti, A. Development of a high-speed current injection and voltage measurement system for electrical impedance tomography based stretchable sensors. Technologies 2017, 5, 48.

3. Yao, A.; Yang, C.L.; Seo, J.K.; Soleimani, M. EIT-based fabric pressure sensing. Comput. Math. Methods Med. 2013, $2013,405325$.

4. Tan, A.M.; Fuss, F.K.; Weizman, Y.; Woudstra, Y.; Troynikov, O. Design of low cost smart insole for real time measurement of plantarpressure. Procedia Technol. 2015, 20, 117-122.

5. Atalay, O.; Kennon, W.; Husain, M. Textile-based weft knitted strain sensors: Effect of fabric parameters on sensor properties, Sensors 2013, 13, 11114-11127.

6. Šafárová, V.; Malachová, K.; Militký, J. Electromechanical analysis of textile structures designed for wearable sensors. In Proceedings of the 16th International Conference on Mechatronics-Mechatronika 2014, Brno, Czech Republic, 3-5 December 2014; pp. 416-422.

7. Atalay, O.; Tuncay, A.; Husain, M.D.; Kennon, W.R. Comparative study of the weft-knitted strain sensors. J. Ind. Text. 2016, 46, 1212-1240, doi:10.1177/1528083715619948.

8. Shopvtechtextile.com. Available online: http://www.shopvtechtextiles.com/assets/images/Fabric\%20Technical\%20Knits\%20pdfs/ $1106001 B \% 20$ Spa cer\%20Fabric\%20B.pdf (accessed on 6 November 2020). 